[Everyday physics]

by Franco Bagnoli,

Dept. of Physics and Astronomy and Center for the Study of Complex Dynamics - University of Florence, Italy

franco.bagnoli@unifi.it - DOI: http://dx.doi.org/10.1051/epn/2016403

\title{
We shoot a bullet vertically. Where will it land?
}

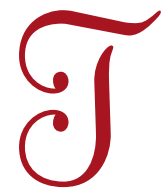

his seems a trivial question, but we must consider the rotation of the Earth. For small elevations (small initial velocities $v_{0}$ ), given the Earth's large radius, the motion of the Earth's surface can be considered to have a constant speed, and the projectile falls back on the point of launch.

But what happens if one increases $v_{0}$ ? Is the landing point affected by the rotation of the Earth?

It is not easy to answer this question without doing the calculations. We can try to make some qualitative considerations, assuming the launching pad to be near the Equator. The projectile maintains its tangential velocity $\omega R_{0}$ (where $R_{0}$ is the radius of the Earth and $\omega$ is the angular velocity of the rotation), but climbing up it "falls behind" with respect to the tangential velocity of the altitude reached, and therefore, during the upward motion, it deviates towards the West (for the same reason why the falling bodies deviate eastward). But on its way down the opposite happens, and it is unclear whether this is enough to have the bullet land at the starting point.

Another consideration is that the projectile motion is similar to the motion of a Foucault pendulum, whose trajectories have the form of rosettes, preceding towards the West. But even this is

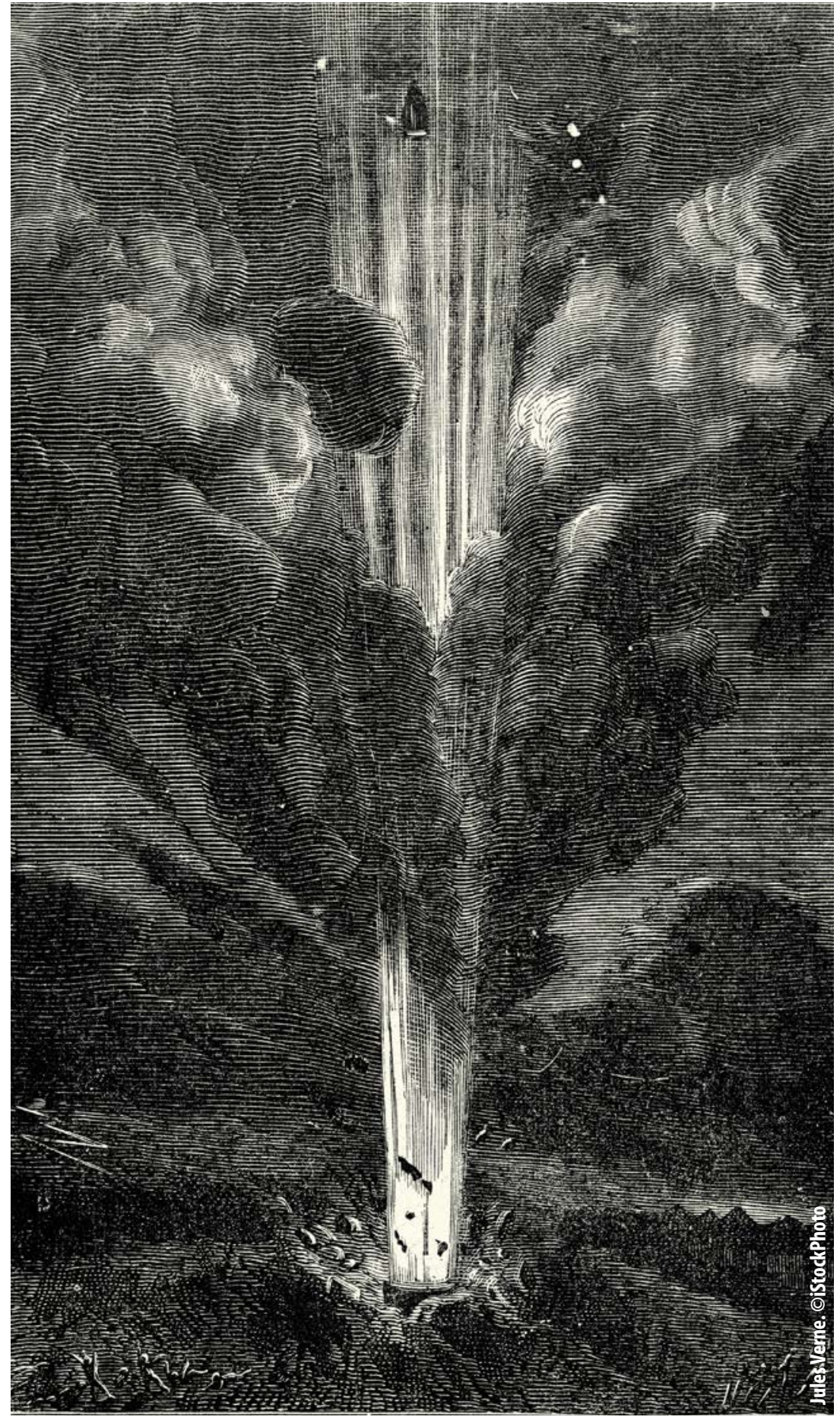


not enough to allow us to decide where the landing spot is. We have to resort to equations, which fortunately are not very difficult to handle (see Box).

Naively one may expect a shift towards the West $[1,2]$, because the Earth will rotate during the flight time of the bullet, 200 seconds in this example. In this naïve picture, the bullet would be assumed to be fired purely vertically, without initial horizontal velocity (in the inertial reference frame). Following this reasoning, the bullet is bound to land West of the launching spot.

The equations show that, indeed, there is a shift to the West. However, it is of a very different nature and magnitude if compared to the naïve result: the tangential velocity of the Earth is $437 \mathrm{~m} / \mathrm{s}$, so, following the naïve picture, in 200 seconds the bullet would land about 87 $\mathrm{km}$ towards the West. In reality it is only $1 \mathrm{~km}$, as shown in the Box.

For more details, see Ref. [2].

\section{References}

[1] https://youtu.be/-9Jp_XCvVto

[2] Franco Bagnoli and Francesco Saverio Cataliotti, Giornale di Fisica vol XII, 103 (2001) doi:10.1393/gdf/i2011-10135-9

\section{BOX}

We proceed in the accelerated reference frame of the Earth. We can neglect the centrifugal force, which does nothing but diminish $g$. Inserting gravity (which we shall consider constant, for not too large elevations, say a maximum of a few kilometers) and the Coriolis force we get the following two equations for the horizontal coordinate $x$ (axis tangential to the Equator, positive direction towards the West) and the elevation coordinate $z$ :

$$
\begin{gathered}
m \ddot{x}=2 m \omega \dot{z}, \\
m \ddot{z}=-m g-2 m \omega \dot{x},
\end{gathered}
$$

where $m$ is the bullet's mass. We can integrate Eq. (1) from 0 (starting point $x(0)=z(0)=0$, with $\dot{z}(0)=v_{0}$ and $\dot{x}(0)=0)$ to $t$, yielding

$$
\dot{x}=2 \omega z,
$$

which tells us that the horizontal motion of the projectile is always directed towards the West. Obviously, the bullet will not fall on the starting point! Substituting Eq. (3) into Eq. (2) we get

$$
\ddot{z}=-g-4 \omega^{2} z
$$

This means that the vertical motion is harmonic, which may be a bit surprising (but obvious if we remember the Foucault pendulum). By replacing the starting conditions in the solution of Eq. (4) we obtain

$$
z(t)=\frac{g}{4 \omega^{2}}[\cos (2 \omega t)-1]+\frac{v_{0}}{2 \omega} \sin (2 \omega t)
$$

which, as anticipated, represents a harmonic oscillation centered in $z(t)=\frac{g}{4 \omega^{2}}$, actually below ground.
To check the plausibility of this solution, let's look at what happens in the limit $\omega \rightarrow 0$ : we get $z(t)=v_{0} t-1 / 2 g t^{2}$, the standard free-fall accelerated motion. From here we can obtain an approximation for the flight time $\tau=\frac{2 v_{0}}{g}$. Substituting Eq. (5) into Eq. (3) and integrating, we obtain

$$
x(t)=\frac{g}{4 \omega^{2}}[\sin (2 \omega t)-2 \omega t]+\frac{v_{0}}{2 \omega}[\cos (2 \omega t)-1]
$$

and in the limit $\omega \rightarrow 0$ we have $x(t)=0$, as expected. In the next (third) order, we obtain

$$
x(t) \simeq \omega\left(v_{0} t^{2}-1 / 3 g t^{3}\right)
$$

Substituting the flight time $\tau=\frac{2 v_{0}}{g}$ we get

$$
x(\tau)=\frac{4 \omega v_{0}^{3}}{3 g^{2}}
$$

Inserting the rotation of the Earth, $\omega=7.27 \cdot 10^{-5} \mathrm{rad} / \mathrm{s}$ (assuming to be at the Equator), and $v_{0}=1000 \mathrm{~m} / \mathrm{s}$ (not unattainable speed with a good gun), we get a maximum height of $50 \mathrm{~km}$ (always neglecting the air resistance!, still small compared to the Earth's radius of $6000 \mathrm{~km}$ ), a flight time $\tau=200 \mathrm{~s}$ and a deviation towards the West of $1 \mathrm{~km}$.

In the presence of the air, the calculation is much more difficult. We cannot assume that the laws of laminar viscous motion apply, since at such speeds the motion will certainly be turbulent. But the bullet should quickly get to such heights that the air density is very low (above $10 \mathrm{~km}$ ). We therefore expect that the bullet will fall at an intermediate distance from the starting point, again towards the West. 